\title{
Coordination on networks with farsighted and myopic agents
}

\author{
Ana Mauleon ${ }^{1} \cdot$ Simon Schopohl ${ }^{1}$. Akylai Taalaibekova ${ }^{2,3}$. \\ Vincent Vannetelbosch ${ }^{4}$
}

Accepted: 11 November 2021 / Published online: 9 January 2022

(C) The Author(s) 2021

\begin{abstract}
We study a coordination game on a fixed connected network where players have to choose between two projects. Some players are moderate (i.e. they are ex-ante indifferent between both projects) while others are stubborn (i.e. they always choose the same project). Benefits for moderate players are increasing in the number of neighbors who choose the same project. In addition, players are either farsighted or myopic. Farsighted players anticipate the reactions of others while myopic players do not. We show that, when all players are farsighted, full coordination among the moderate players is reached except if there are stubborn players for both projects. When the population is mixed, the set of stable strategy profiles is a refinement of the set of Nash equilibrium strategy profiles. In fact, turning myopic players into farsighted ones eliminates gradually the inefficient Nash equilibria. Finally, we consider a social planner who can improve coordination by means of two policy instruments: adding links to the network (socialization) and/or turning myopic players into farsighted ones (education).
\end{abstract}

Keywords Networks $\cdot$ Coordination problems $\cdot$ Stubborn players $\cdot$ Farsighted players $\cdot$ Stability

JEL Classification A14 · C70 - D20

$凶$ Vincent Vannetelbosch

vincent.vannetelbosch@uclouvain.be

Ana Mauleon

ana.mauleon@usaintlouis.be

Akylai Taalaibekova

akylai.taalaibekova@uclouvain.be

1 CEREC and CORE, UCLouvain, Louvain-la-Neuve, Belgium

2 CORE, UCLouvain, Louvain-la-Neuve, Belgium

3 CES, Université Paris 1 Panthéon-Sorbonne, Paris, France

4 CORE and CEREC, UCLouvain, Louvain-la-Neuve, Belgium 


\section{Introduction}

Coordination problems play an important role in social and economic interactions. Partners, family and friends coordinate what to eat, how to spend the evening or where to go on vacation. Researchers coordinate with their coauthors time and date of meetings and the splitting of tasks. Firms active in research and development or joint production partnerships often need to coordinate on the choice of compatible technologies. Countries involved in trade and political alliances must agree on conventions such as a communication language. In most of those coordination problems, agents make choices positively influenced by their neighbors (friends, collaborators, ...) in the network. ${ }^{1}$

In this paper we address fundamental questions about coordination games in networks. Does farsightedness solve the coordination problem? Do stubborn agents impede coordination in the presence of farsighted agents? Do we improve coordination by turning myopic agents into farsighted ones? Can a social planner reach full coordination by stimulating socialization (adding links to the network) or education (making myopic agents becoming farsighted)?

To answer these questions we study a coordination game on a fixed connected network. Players have to choose between two projects. Some players are moderate (i.e. they are ex-ante indifferent for both projects) while others are stubborn players (i.e. they always choose the same project independently of what others do). Benefits are supposed to be increasing in the number of friends or neighbors who choose the same project, conditionally on the project being successful. A project is said to be successful if it is adopted by enough people within the whole society. In addition of being either moderate or stubborn, each player can be either myopic or farsighted. In contrast to myopic players, farsighted players anticipate that others might react to their actions and take into account the sequence of reactions that results from their own actions in the network.

We first solve the coordination game when all players are farsighted. We propose the concept of farsightedly stable strategy profile for determining the outcome of the game. A strategy profile is farsightedly stable if there is no farsighted improving path emanating from it. ${ }^{2}$ A farsighted improving path is a sequence of strategy profiles where each time one player chooses an action based on the improvement the end strategy profile offers her relative to the current strategy profile. We show that there always exists a farsightedly stable strategy profile. Suppose there are no stubborn players or only stubborn players for a single project. Then, farsightedness leads to full coordination among the whole society, even though there is a large multiplicity of inefficient Nash equilibria. Once there are stubborn players for both projects, farsightedness does not always overcome the issue of miscoordination. Intuitively, farsighted players may fail to influence other moderate players if the only channels for influencing them go

\footnotetext{
1 Jackson (2008) and Goyal (2007) provide a comprehensive introduction to the theory of social and economic networks. Mauleon and Vannetelbosch (2016) give an overview of the solution concepts for solving network formation games.

2 See Chwe (1994); Dutta et al. (2005); Herings et al. (2009, 2019); Page and Wooders (2009); Ray and Vohra $(2015,2019)$ among others for alternative notions of farsightedness.
} 
through stubborn players. Nevertheless, we provide conditions to guarantee that all moderate players fully coordinate on the same project.

We next consider the case of a mixed population consisting of myopic and farsighted players. Indeed, recent experiments provide evidence in favor of a mixed population consisting of both myopic and farsighted individuals (see Kirchsteiger et al. 2016). We propose the concept of myopic-farsighted stable strategy profile for determining the outcome of the game. A strategy profile is myopic-farsighted stable if there is no myopic-farsighted improving path emanating from it. A myopic-farsighted improving path is a sequence of strategy profiles that can emerge when farsighted players choose an action based on the improvement the end strategy profile offers them relative to the current strategy profile while myopic players choose an action based on the improvement the resulting strategy profile offers them relative to the current one. We show that when all players are myopic the set of stable strategy profiles coincides with the set of Nash equilibria of the coordination game. The set of stable strategy profiles weakly decreases when a myopic player becomes farsighted. Hence, the set of myopic-farsighted stable strategy profiles when moderate players are both myopic and farsighted is a refinement of the set of Nash equilibrium strategy profiles. In fact, turning myopic players into farsighted ones eliminates gradually the inefficient Nash equilibria. We then look at the impact of stubbornness and at what happens for some specific networks (complete, star and line networks).

Finally, we consider a social planner who can improve coordination by means of two policy instruments: adding links to the network (socialization) and/or turning myopic players into farsighted ones (education). In the case of regular networks, especially circle networks, both instruments are substitutes when all players are moderate. However, in the presence of stubborn players, turning myopic players into farsighted ones without adding links cannot lead to full coordination among the moderate players.

One motivation for our model is the adoption of new technologies. Agents choose whether to adopt a new technology or keep the old one, such as in social media, where the benefit of the technology is increasing in the number of friends or neighbors that adopt the same technology. For being successful or profitable, the technology needs to be adopted by a certain mass of customers; otherwise, it probably disappears in the long run. In addition, we often encounter within the population agents who stick no matter what to the old technology while others are slavishly devoted to the new technologies, like nerds. Another example is the cooperation in R\&D between researchers or firms. Researchers can be involved in a network of R\&D collaborations, where the links can be interpreted as previous cooperation on a joint project. Given the network, the researchers can cooperate into new projects that require the collaboration of several researchers. While some researchers might be interested in participating into the new projects, other researchers could be reluctant to participate in some new project. In order to be successful, all required researchers should collaborate in the joint project. The collaboration with a higher number of researchers reduce more the effort the researcher has to deploy for her research. Other examples include situations involving 
social influence regarding the choice by the society between alternative projects for the development of the country. ${ }^{3}$

We now turn to the related literature. In our coordination game played on a fixed network, players have incentives to coordinate with their neighbors. But, there are situations where the opposite happens. Bramoullé (2007) studies anti-coordination games and defines frustration as a function of neighbors who play the same action and neighbors who choose a different action. Frustration is the highest in the complete network and the lowest in bipartite networks. Bramoullé et al. (2004) go one-step further by endogenizing the network formation. Jackson and Watts (2002a) also look at coordination games on networks, but rather focus on repeated interactions. In each period, first a randomly selected link can be added or deleted, next a randomly chosen player can adjust her action. With a small probability a player makes a mistake which might induce other players to modify their actions later on. All players are myopic and are ex-ante indifferent between the different alternatives. For solving this game, they use the concept of stochastic stability. They find that the endogenous network not only has implications for the interaction pattern that emerges, but it also has a significant impact on the play in the coordination game relative to what would arise if the same interaction network was exogenous. Goyal and Vega-Redondo (2005) also study a coordination game which is repeatedly played on networks where players can choose between two different options. Players can revise their strategy in each period with a certain probability. In their model the links between players are paid only by one player. The authors show that depending on the costs for a link players either coordinate on the risk-dominant action or on the efficient action.

Morris (2000) considers an infinite population of players that revise their actions according to deterministic best response dynamics. Each player must choose between one of two actions ( 0 and 1$)$ to play against a finite subset of neighbors. Preferences take a local-aggregate form and it is assumed that there exists a critical number q between 0 and 1 such that action 1 is a best response for a player if at least a proportion $\mathrm{q}$ of his neighbors plays 1 . Contagion occurs if one action spreads from a finite set of players to the whole population. Morris (2000) characterizes the contagion threshold such that contagion occurs. In a related paper, Jackson and Storms (2019) analyze a game in which each player can either adopt the behavior of her neighbors or not. Following Morris (2000), it is assumed that if a certain amount of neighbors of a player choose to adopt some behavior, it becomes beneficial for this player to adopt this behavior as well. There can be multiple different conventions in which players in some parts of the network adopt a behavior while players in other parts of the network do not. They define communities to be groups of players who behave the same as each other in every convention. They characterize the behavioral communities in some random graphs as a function of how strongly the benefits of adopting the behavior depend on others' behaviors.

\footnotetext{
3 In Switzerland, people had to choose in 2018 between being candidate for organizing the Winter Olympic Games 2026 or not. The no-option reverts to allocate the proposed budget to more durable activities. The no-option won by $53,98 \%$ and from the analysis of the votes and polls it was observed that most people from the cities were voting against except those having strong ties with the ski resorts (Le Nouvelliste, 10/6/2018).
} 
Recently, Leister et al. (2021) study a coordination game among agents on a network who choose whether or not to adopt a new technology in an uncertain environment. In their model, the total value an agent receives from adopting the technology increases in the technology's underlying value (the state) and in its adoption by neighbors. They propose an algorithm that fully characterizes the equilibrium coordination sets within the network, and they provide an exact condition for which a single coordination set exists in the network. While they explore the role of uncertainty and the network's architecture in determining who coordinates their adoption choices with whom, we examine the impact of a heterogeneous population consisting of myopic and farsighted players as well as moderate and stubborn players on the coordination outcome.

Closest to our work, but without the network aspect, is the work of Sakovics and Steiner (2012). They focus on a continuum of players where each player can decide to either invest into a project or abstain. The project has an investment threshold and is only successful if enough players invest. Players receive a noisy signal about this threshold. The authors show that depending on the noise in the signal there can be miscoordination, while if the noise is small players coordinate their actions. ${ }^{4}$

We further the literature by considering the impact of a mixed population along two dimensions (moderate versus stubborn players, farsighted versus myopic players) on the coordination problem. We propose the notion of myopic-farsighted stable strategy profiles and show that it refines the concept of Nash equilibrium. In addition, our analysis reveals how crucial it might be to have precise knowledge of the social and economic network for implementing efficiently policies for improving coordination among the interacting agents.

The structure of the paper is as follows: In Sect. 2 we describe the coordination game played on a fixed network. In Sect. 3 we define the concept of farsightedly stable strategy profiles and we prove its existence. We then characterize the set of stable strategy profiles in the presence of either only moderate players, or moderate players with stubborn players for a single project, or moderate players together with stubborn players of both types. In Sect. 4 we introduce myopic players together with farsighted ones, and possibly stubborn players. We propose the concept of myopicfarsighted stable strategy profiles and we analyze the impact of farsightedness and stubbornness. In Sect. 5 we look at the role of the social planner whose objective is to improve coordination within the whole network. In Sect. 6 we conclude. All proofs are relegated to the appendix.

\section{Coordination problems}

\subsection{Networks}

Let $N=\{1, \ldots, n\}$ be a finite set of players who are located on a fixed connected network $g$. A network $g$ is a list of which pairs of players are linked to each other and $i j \in g$ indicates that $i$ and $j$ are linked under $g$. A path in a network $g$ between $i$ and

\footnotetext{
4 Another related strand of literature where we observe similar network effects are local public good models that do analyze the provision of public goods in networks (see e.g. Bramoullé and Kranton 2007; Allouch 2015).
} 
$j$ is a sequence of players $i_{1}, \ldots, i_{K}$ such that $i_{k} i_{k+1} \in g$ for each $k \in\{1, \ldots, K-1\}$ with $i_{1}=i$ and $i_{K}=j$. A network $g$ is connected if for all $i \in N$ and $j \in N \backslash\{i\}$, there exists a path in $g$ connecting $i$ and $j$. Let $g^{S}$ be the set of all subsets of $S \subseteq N$ of size 2, so $g^{N}$ is the complete network. Let $N(g)=\{i \mid$ there is $j$ such that $i j \in g\}$ be the set of players who have at least one link in the network $g$. Let $N_{i}(g)=\{j \in N \mid i j \in g\}$ be the set of neighbors of player $i$ in $g$. The degree of player $i$ in network $g$, denoted $d_{i}(g)$, is the cardinality of $i$ 's set of neighbors, $d_{i}(g)=\# N_{i}(g){ }^{5}$ A player who has only one neighbor is called a leaf. A star network is a network such that there exists some player $i$ (the center) who is linked to every other player $j \neq i$ (the peripherals) and that contains no other links (i.e. $g$ is such that $N_{i}(g)=N \backslash\{i\}$ and $N_{j}(g)=\{i\}$ for all $j \in N \backslash\{i\})$. A line network is a connected network where each player $i \in N \backslash\{j, k\}$ has two links while players $j$ and $k$ are leaves.

\subsection{A coordination game played on a fixed network}

The society consists of $n$ players located on a fixed network who have to choose between two projects $p$ or $q$. A project $r \in\{p, q\}$ is said to be successful if at least $t^{r}$ players choose this project. We call $t^{r}$ the support threshold of project $r \in\{p, q\}$, $0<t^{r} \leq n$ and $t^{r} \in \mathbb{N}$. Let $t=\left(t^{p}, t^{q}\right)$. Some players are moderate, while others are stubborn. Each moderate player chooses one project or action $a_{i} \in\{p, q\}$. Let $N_{m}=$ $\{1, \ldots, m\}$ be the set of moderate players. A stubborn player for project $r \in\{p, q\}$ always chooses project $r$ whatever the choice of other players and the thresholds. Let $N_{r}$ be the set of stubborn players for project $r, r \in\{p, q\}$. Thus, if $i \in N_{r}$ then $a_{i}=r$. Let $N_{p} \cup N_{q}=\{m+1, \ldots, n\}$. Let $a=\left(a_{1}, \ldots, a_{n}\right)$ be a strategy profile and $A$ be the set of all possible strategy profiles. ${ }^{6}$ The set of players $N=\{1, \ldots, n\}$ is simply $N_{m} \cup N_{p} \cup N_{q}{ }^{7}$

Given a strategy profile $a \in A$, let $n_{i}(a)=\#\left\{j \in N \mid a_{i}=a_{j}\right\}$ be the total number of players who choose the same project as player $i$ (stubborn players as well as player $i$ included), and let $\bar{n}_{i}(g, a)=\#\left\{j \in N \mid a_{i}=a_{j}\right.$ and $\left.i j \in g\right\}$ be the number of neighbors (stubborn players included) of player $i$ in the network $g$ who choose the same project as player $i$. Given some network $g$, some thresholds $\left(t^{p}, t^{q}\right)$ and some strategy profile $a \in A$, the utility function $U_{i}$ of a moderate player $i \in N_{m}$ is given by the following expression:

$$
U_{i}\left(g ; a_{i}, a_{-i}\right)= \begin{cases}-c_{i} & \text { if } n_{i}\left(a_{i}, a_{-i}\right)<t^{a_{i}}, \\ b_{i}+\phi_{i}\left(\bar{n}_{i}(g, a)\right) & \text { if } n_{i}\left(a_{i}, a_{-i}\right) \geq t^{a_{i}},\end{cases}
$$

where $c_{i} \geq 0$ is player $i$ 's loss from investing into an unsuccessful project while $b_{i}+\phi_{i}\left(\bar{n}_{i}(g, a)\right)$ is player $i$ 's benefit from a successful project. This benefit is composed of two parts: a fixed one $\left(b_{i}>0\right)$ and a variable one $\left(\phi_{i}\left(\bar{n}_{i}(g, a)\right) \geq 0\right)$ which depends

\footnotetext{
5 Throughout the paper we use the notation $\subseteq$ for weak inclusion and $\varsubsetneqq$ for strict inclusion. Finally, \# will refer to the notion of cardinality.

6 Notice that strategy profiles where some stubborn player for one project would choose the other project are excluded from the set of all possible strategy profiles $A$.

7 For a $n \times 1$ vector $a$ we denote by $a_{-i}$ the vector of size $n-1$ with the $i$-th entry removed and we use $a_{-i, j}$ to denote the vector in which the $i$-th and the $j$-th elements have been deleted.
} 
Fig. 1 Network with four moderate players $(1,2,3,4)$ and three stubborn players $(5,6,7)$

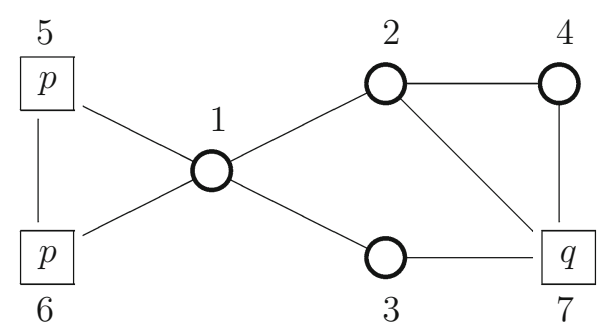

on the action chosen by the neighbors of player $i$. The function $\phi_{i}$ is increasing with the number of neighbors who choose the same project as $i$ and it captures the fact that players may have different interests in cooperating with their neighbors. So, an investment of player $i$ into an unsuccessful project $\left(n_{i}\left(a_{i}, a_{-i}\right)<t^{a_{i}}\right)$ gives her a negative utility $\left(-c_{i}\right)$, while an investment into a successful project $\left(n_{i}\left(a_{i}, a_{-i}\right) \geq t^{a_{i}}\right)$ yields her a positive utility. Furthermore, in the latter case, the utility of player $i$ is increasing with the number of neighbors who choose the same project. ${ }^{8}$

Notice that, according to the utility function, the threshold depends on global adoption whereas player $i$ 's benefit depends on local adoption by her neighbors. ${ }^{9}$ Many applications in real life match this type of utility function. For instance social media platforms become attractive for adoption once a significant amount of users and/or social influencers are using them. In other words, an individual user registers on the new platform only when the platform has been globally adopted. At the same time, the benefits that an individual user derives from the platform depend on the number of friends with whom she communicates and shares information through the platform; i.e., the local adoption among her social network. ${ }^{10}$

Let us point out some properties of this utility function. In the case player $i$ chooses a successful project, her utility increases if some neighbor $j$ switches to her project: $U_{i}\left(g ; a_{i}, a_{j}, a_{-i, j}\right)>U_{i}\left(g ; a_{i}, a_{j}^{\prime}, a_{-i, j}\right)$ if $a_{j}^{\prime} \neq a_{j}=a_{i}, j \in N_{i}(g)$ and $n_{i}\left(a_{i}, a_{j}, a_{-i, j}\right) \geq t^{a_{i}}$. The utility of player $i$ does not change if her neighbor, player $j$, changes her action but the project chosen by player $i$ remains unsuccessful: $U_{i}\left(g ; a_{i}, a_{-i}\right)=U_{i}\left(g ; a_{i}, a_{-i}^{\prime}\right)$ for any $a_{-i}$ and $a_{-i}^{\prime}$ if $n_{i}\left(a_{i}, a_{-i}\right)<t^{a_{i}}$ and $n_{i}\left(a_{i}, a_{-i}^{\prime}\right)<t^{a_{i}}$.

From now on we only consider projects that can be successful: $\# N_{m}+\# N_{r} \geq t^{r}$ for $r \in\{p, q\}$. It ensures that each project $r$ can be successful because there are not too many stubborn players for the other project such that the threshold of project $r$ cannot be reached even if all stubborn players for project $r$ and all moderate players invest into project $r$.

\footnotetext{
8 For simplicity, we assume that a stubborn player for project $r \in\{p, q\}$ always chooses project $r$ whatever the choice of other players and the thresholds. Such behavior would emerge endogenously by simply assuming that a stubborn player $i$ choosing the alternative project obtains a disutility of $-c_{i}-\bar{c}(\bar{c}>0)$.

9 The preferences considered in Morris (2000); Jackson and Storms (2019) and other closely related papers are such that both the threshold and the individual benefits depend on local adoption.

10 Other applications could fit the opposite utility function where the threshold depends on local adoption and the individual benefit depends on global adoption. The study of such situations is beyond the scope of this paper.
} 
In Fig. 1 we illustrate a situation with seven players located on a network who have to decide on investing into alternative projects $p$ or $q$. Players with a round node are moderate players, while players with a square node are stubborn for the project written in the square. In this example there are two stubborn players for project $p$, namely players 5 and 6. Player 7 is stubborn for project $q$ while players 1, 2, 3 and 4 are moderate. Suppose players 1 and 2 choose $p$ while players 3 and 4 choose $q$. Then, $a=(p, p, q, q, p, p, q)$.

\section{Farsighted agents}

\subsection{Farsightedly stable strategy profiles}

Typically, coordination games are solved using the concept of Nash equilibrium. A strategy profile $a^{*} \in A$ is a Nash Equilibrium if $U_{i}\left(g ; a_{i}^{*}, a_{-i}^{*}\right) \geq U_{i}\left(g ; a_{i}, a_{-i}^{*}\right)$ for all $i \in N_{m}$, for all $a_{i} \in\{p, q\}$. Let $\mathcal{N}(g, t)$ denote the set of all Nash Equilibria.

Assume now that players are farsighted. Farsighted players forecast how others might react to their actions and take into account the sequence of reactions that results from their own actions in the network. A farsighted improving path is a sequence of strategy profiles that can emerge when farsighted players choose an action based on the improvement the end strategy profile offers them relative to the current strategy profile and where each strategy profile in the sequence differs from the preceding one in that only one action of one of the players is modified.

Definition 1 Consider $(g, t)$ as given. A farsighted improving path from a strategy profile $a \in A$ to a strategy profile $a^{\prime} \in A$ is a finite sequence of strategy profiles $a^{0}, \ldots, a^{L} \in A$ with $a^{0}=a, a^{L}=a^{\prime}$ and $a^{j} \neq a^{k}$ for all $j, k \in\{1, \ldots, L-1\}$ such that for every $\ell \in\{0, \ldots, L-1\}$, we have $a_{i}^{\ell+1} \neq a_{i}^{\ell}$ and $a_{-i}^{\ell+1}=a_{-i}^{\ell}$ for some $i \in N$, and $U_{i}\left(a^{L}\right)>U_{i}\left(a^{\ell}\right)$.

If there exists a farsighted improving path from the strategy profile $a$ to $a^{\prime}$ we write $a \rightarrow a^{\prime}$. The set of all strategy profiles that can be reached from $a$ by a farsighted improving path is denoted by $f(a)=\left\{a^{\prime} \in A \mid a \rightarrow a^{\prime}\right\}$. Our notion of farsighted improving path is similar to the one first developed by Jackson (2008) and Herings et al. (2009) for network formation.

Example 1 Consider the network depicted in Fig. 2. All players are farsighted. Players 6 and 7 are stubborn while all other players are moderate. Both projects $p$ and $q$ have a low threshold: $t^{p}=t^{q}=1$. Start with the profile $a^{0}=(p, p, p, p, p, p, q)$ where player 5 is coordinating with one of her two neighbors. From this profile there is a farsighted improving path initiated by player 5 . First, player 5 chooses project $q$ instead of $p$ which yields to the profile $a^{1}=(p, p, p, p, q, p, q)$. In this profile $a^{1}$ player 3 has now an incentive to deviate to project $q$ as well, because two of her three neighbors have selected project $q$. This yields to the profile $a^{2}=(p, p, q, p, q, p, q)$. We observe that in $a^{2}$ player 5 is coordinating with both of her neighbors. Since she is farsighted, she had the incentive to start the improving path, taking into account that player 3 will follow her example and deviate from project $p$ to $q$ afterwards. This 
Fig. 2 Network with five moderate players $(1,2,3,4,5)$ and two stubborn players $(6,7)$

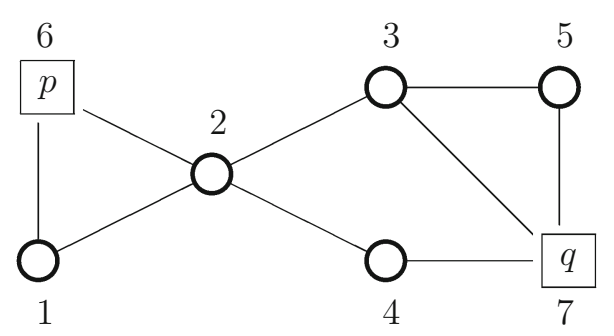

makes player 5 in profile $a^{2}$ better off than in $a^{0}$, even though she did not immediately (in $a^{1}$ ) benefit from her initial change.

Farsighted players anticipate the changes of the other players and compare their current utility with the utility they would get at the end of the improving path. Nash equilibrium requires strategy profiles to be immune to immediate deviations and does not capture that farsighted players anticipate the actions of other players and consider the end strategy profile that their deviations may lead to. To be stable, a strategy profile should be immune to deviations of farsighted players. These considerations lead to the following definition of farsightedly stable strategy profiles.

Definition 2 Consider $(g, t)$ as given. A strategy profile $a \in A$ is farsightedly stable if it satisfies $f(a)=\emptyset$.

A strategy profile $a \in A$ is farsightedly stable if there is no farsighted improving path leaving $a$. Let $Z(g, t)=\{a \in A \mid f(a)=\emptyset\}$ be the set of farsightedly stable strategy profiles. ${ }^{11}$

Example 2 We reconsider the network depicted in Fig. 2 where players 6 and 7 are stubborn while all other players are moderate. We still assume that $t^{p}=t^{q}=1$. Take $a^{*}=(p, p, q, q, q, p, q)$ where players 1 and 2 choose $p$ as the stubborn player 6 , while players 3,4 and 5 choose project $q$ as the stubborn player 7 . We now argue that there are no farsighted improving path emanating from $a^{*}$. That is, $f\left(a^{*}\right)=\emptyset$. From any $a \in A$ such that $a_{5}=q$ player 5 will never change her action since there is no $a^{\prime} \in A$ where she would be strictly better off. Similarly, for player 1 when choosing project $p$ and for player 4 when choosing project $q$. Anticipating that player 5 will never switch her action, player 3 cannot be better off by choosing $p$ instead of $q$. Hence, player 3 will stick to project $q$. Finally, given that no other player will initiate a move from $(p, \cdot, q, q, q, p, q)$ and that player 2 is indifferent between $(p, p, q, q, q, p, q)$ and $(p, q, q, q, q, p, q)$, player 2 will not deviate from $(p, p, q, q, q, p, q)$. So, $f\left(a^{*}\right)=\emptyset$ and $(p, p, q, q, q, p, q)$ is a farsightedly stable strategy profile.

11 Similarly, Jackson (2008) defines the concept of farsightedly pairwise stable networks. Farsightedly pairwise stable networks are the networks from which no farsighted improving path is emanating of it. 


\subsection{Existence of a farsightedly stable strategy profile}

We now show that there always exists a farsightedly stable strategy profile, i.e., $Z$ is never empty, and we provide a method to find a farsightedly stable strategy profile. Starting from an arbitrary profile $a$, we can start writing the decision-making process of a player along a farsighted improving path as a tree. At each node one player decides whether she wants to stay with her current action or wants to deviate to another action. Since players can change their action many times along a farsighted improving path, the decision order of players can be neglected. The order only decides which profile is reached, but if there is a farsighted improving path from $a$ when players decide in the order 1 to $m$, then there is also one for every other decision order. This is due to the fact that players can repeatedly change their actions. Still, it is not possible to illustrate an entire farsighted improving path as a tree, because there is an infinite number of nodes. Again, the reason is the possibility of repeated deviations from each player.

The following lemma allows us to write down the decision-making process as a finite tree when all players are farsighted.

Lemma 1 If there exists a farsighted improving path from profile a to profile $a^{\prime}$ then there also exists a farsighted improving path from a to $a^{\prime}$ in which each player changes her action at most once.

Lemma 1 implies that instead of considering all farsighted improving paths, we can focus our attention on farsighted improving paths in which each player changes her action once or not at all. To find a farsightedly stable strategy profile we formulate a game tree. First, we need to fix a decision order among the moderate players, without loss of generality $(1, \ldots, m)$ and we select an arbitrary profile $a^{0}$. Then, we can start to create the decision tree. In the first node player 1 decides if she wants to stay with her action $a_{1}^{0}$ or if she wants to deviate to any other action. In case of indifference we assume she stays. If she deviates, we reach a new profile $a^{1}$ with $a_{1}^{0} \neq a_{1}^{1}$ and $a_{-1}^{0}=a_{-1}^{1}$. In that case we remove player 1 from the decision order and we start from the beginning of the updated order, in this case with player 2. Player 2 then faces the same choice, staying in $a^{1}$ or deviating. If she deviates we move to $a^{2}$, remove player 2 from the decision order and start from the beginning of the order. If player $i$ does not deviate, but stays in profile $a^{x}$, we move to the next player in the decision order and letting her decide whether she wants to deviate from $a^{x}$. When player $i$ does not change her decision, she stays in the decision order and can revisit her choice once another player has deviated. We reach a stable strategy profile either when we reach the end of the decision order and no player wants to deviate or when there are no players left in the decision order. To illustrate this game tree, we introduce the following notation. Let $o$ be the decision order of the moderate players, without loss of generality $(1, \ldots, m)$ and $o \backslash i$ implies that player $i$ is removed from the decision order, i.e. $o \backslash i=(1, \ldots, i-1, i+1, \ldots m)$. We generate the game tree for $\Gamma\left(a^{0}, o\right)$, i.e. starting with profile $a^{0}$ and order $o$ as shown in Fig. 3. $\Gamma\left(a^{k}, \emptyset\right)$ gives the utility $U_{i}\left(a^{k}\right)$ to player $i$ where $\Gamma\left(a^{k}, \emptyset\right)$ represents the profile reached once all moderate players have been removed from the decision order or they don't want to deviate. 


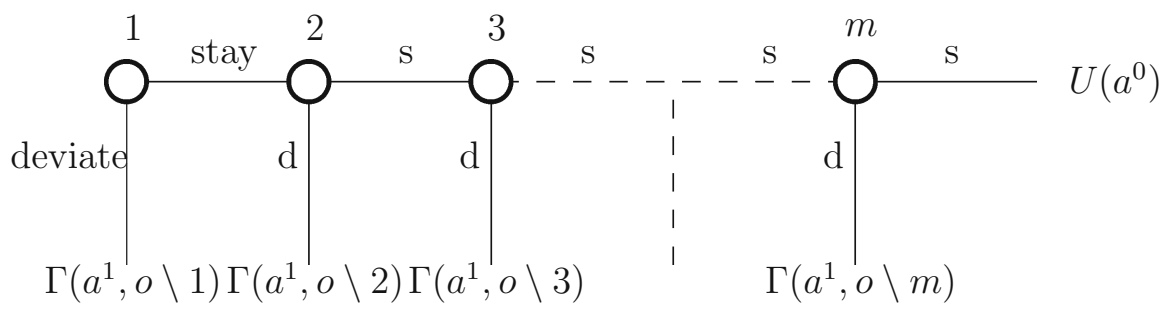

Fig. 3 Game tree of $\Gamma\left(a^{0}, o\right)$

By construction, the finite game tree of $\Gamma\left(a^{0}, o\right)$ shows all the possible improving paths emanating from $a^{0}$ in which each farsighted moderate player deviates at most once. Together with Lemma 1 this yields the following result.

Theorem 1 The subgame perfect Nash equilibrium of the game $\Gamma\left(a^{0}, o\right)$ as defined above is a farsightedly stable profile.

Theorem 1 implies that there always exists at least one farsightedly stable strategy profile, i.e. the set of farsightedly stable profiles is non-empty. Starting from different profiles $a^{0}$ or using different orders $o$ can result in different farsightedly stable strategy profiles.

Example 3 We reconsider the network depicted in Fig. 2 where players 6 and 7 are stubborn while all other players are moderate. We still assume that $t^{p}=t^{q}=1$. We can start from the profile $a^{o}=(p, p, p, p, p, p, q) \in A$ and the decision order $o=(1, \ldots, 5)$. Starting from profile $a^{0}$ players 1 to 4 have no incentive to deviate, but player 5 does. She will select project $q$ even though this action does not provide her an immediate improvement, because she is anticipating that afterwards player 3 will deviate to project $q$ in the game $\Gamma((p, p, p, p, q, p, q),(1, \ldots, 4))$. After players 5 and 3 have switched to $q$ we reach the game $\Gamma((p, p, q, p, q, p, q),(1,2,4))$ where none of the players 1,2 or 4 have any incentive to change her action. So, the profile ( $p, p, q, p, q, p, q)$ is farsightedly stable.

If we start from the profile $(q, q, q, q, q, p, q)$ no player will deviate and so it is farsightedly stable.

\subsection{No stubborn agents}

Suppose there are no stubborn players (i.e. $N=N_{m}$ ) and all moderate players are farsighted. Remember that $t$ is such that $\# N_{m}+\# N_{r} \geq t^{r}$ for $r \in\{p, q\}$. Hence, $\# N_{m} \geq t^{r}$ for $r \in\{p, q\}$. Farsighted moderate players will then choose the same project whatever the connected network.

Proposition 1 Suppose there are no stubborn players and all moderate players are farsighted. Then, $Z(g, t)=\left\{a \in A \mid a_{i}=a_{j}\right.$ for all $\left.i, j \in N\right\}$.

When players are farsighted they are able to coordinate on the same project and all inefficient Nash equilibria become unstable. Thus, without stubborn players, farsightedness solves the coordination problem. 


\subsection{Only one type of stubborn agents}

Suppose there is only one type of stubborn players, without loss of generality for project $p$ (i.e. $N=N_{m} \cup N_{p}$ ), and all moderate players are farsighted. Remember that $t$ is such that $\# N_{m}+\# N_{p} \geq t^{p}$. All farsighted moderate players will then choose project $p$ whatever the connected network.

Proposition 2 Suppose there are only stubborn players for project $p$ and all moderate players are farsighted, $N=N_{m} \cup N_{p}$. Then, $Z(g, t)=\left\{a \in A \mid a_{i}=p\right.$ for all $i \in N\}=\{(p, p, \ldots, p, p)\}$.

Thus, with only one type of stubborn players, farsightedness still solves the coordination problem.

\subsection{Both types of stubborn agents}

Suppose now that there are stubborn players for project $p\left(N_{p} \neq \emptyset\right)$ and stubborn players for project $q\left(N_{q} \neq \emptyset\right)$ as well as moderate players (i.e. $\left.N=N_{m} \cup N_{p} \cup N_{q}\right)$. Remember that all moderate players are farsighted and the threshold $t$ is such that $\# N_{m}+\# N_{r} \geq t^{r}$ for $r \in\{p, q\}$. We provide first a condition to guarantee that all moderate players fully coordinate on the same project, namely the one chosen by the larger group of stubborn players.

Proposition 3 If \# $\left(N_{i}(g) \cap N_{p}\right)>\#\left(N_{i}(g) \cap N_{q}\right)$ for all $i \in N_{m}$, then the strategy profile $a \in A$ with $a_{i}=p$ for all $i \in N_{m}$ is the unique farsightedly stable strategy profile.

We provide next a condition to guarantee that a subset of the moderate players coordinate on the same project.

Proposition 4 Suppose that $t^{r} \leq \# N_{r}+1$ for $r \in\{p, q\}$. If some moderate player $j \in N_{m}$ is such that (i) \# $\left(N_{j}(g) \cap N_{p}\right)>\#\left(N_{j}(g) \cap N_{q}\right)$ and (ii) on all paths from $j$ to $i \in\left\{l \in N_{m} \mid \#\left(N_{l}(g) \cap N_{q}\right)>\#\left(N_{l}(g) \cap N_{p}\right)\right\}$ there is some stubborn player $k \in N_{p}$, then player $j$ chooses $a_{j}=p$ in any farsightedly stable strategy profile.

Proposition 4 tells us that a moderate player $j$ will choose project $p$ if she is (i) neither influenced by stubborn players for project $q$, (ii) nor by moderate players who are themselves influenced by more stubborn players for project $q$ than for project $p$. In fact, all moderate players on the paths from $j$ up to reaching a first stubborn player for project $p$ (if any) will choose project $p$ since they also satisfy the condition of Proposition 4. Hence, all moderate players within the local network of player $j$ will coordinate on project $p .^{12}$

\footnotetext{
12 Under the conditions of Proposition 4, there is some stubborn player for project $p$ in every path in the network connecting the subset of moderate players satisfying the conditions of Proposition 4 and any other moderate player under the influence of stubborn players for project $q$. Hence, these stubborn players for project $p$ behave as essential intermediaries (see Manea 2021) and are able to enforce coordination on project $p$ among the subsets of moderate players that are influenced by them.
} 
Notice that if there is some $j \in N_{m}$ such that $\#\left(N_{j}(g) \cap N_{p}\right)>\#\left(N_{j}(g) \cap N_{q}\right)$ and on all paths from $j$ to $i \in\left\{l \in N_{m} \mid \#\left(N_{l}(g) \cap N_{q}\right)>\#\left(N_{l}(g) \cap N_{p}\right)\right\}$ there is some stubborn player $k \in N_{p}$, and there is some moderate player $j^{\prime} \in N_{m}$ such that $\#\left(N_{j^{\prime}}(g) \cap N_{q}\right)>\#\left(N_{j^{\prime}}(g) \cap N_{p}\right)$ and on all paths from $j^{\prime}$ to $i \in\left\{l \in N_{m} \mid\right.$ $\left.\#\left(N_{l}(g) \cap N_{p}\right)>\#\left(N_{l}(g) \cap N_{q}\right)\right\}$ there is some stubborn player $k \in N_{q}$, then both projects $p$ and $q$ will coexist and be adopted by moderate players in any farsightedly stable strategy profile.

\section{Myopic with farsighted agents}

Suppose now that we face a mixed population of moderate players in terms of their degree of farsightedness. That is, moderate players can be either farsighted or myopic. We denote by $M$ the set of myopic players and by $F$ the set of farsighted players $\left(N_{m}=F \cup M\right)$. Either set ( $M$ or $F$ ) is allowed to be empty. A myopic-farsighted improving path is a sequence of strategy profiles that can emerge when farsighted players choose an action based on the improvement the end strategy profile offers them relative to the current strategy profile while myopic players choose an action based on the improvement the resulting strategy profile offers them relative to the current one. Each strategy profile in the sequence differs from the preceding one in that only one action of one of the players is modified.

Definition 3 Consider $(g, t)$ as given. A myopic-farsighted improving path from a strategy profile $a \in A$ to a strategy profile $a^{\prime} \in A$ is a finite sequence of strategy profiles $a^{0}, \ldots, a^{L} \in A$ with $a^{0}=a, a^{L}=a^{\prime}$ and $a^{j} \neq a^{k}$ for all $j, k \in\{1, \ldots, L-1\}$ such that for every $\ell \in\{0, \ldots, L-1\}$, we have $a_{i}^{\ell+1} \neq a_{i}^{\ell}$ and $a_{-i}^{\ell+1}=a_{-i}^{\ell}$ for some $i \in N$, and $U_{i}\left(a^{\ell+1}\right)>U_{i}\left(a^{\ell}\right)$ if $i \in M$ or $U_{i}\left(a^{L}\right)>U_{i}\left(a^{\ell}\right)$ if $i \in F$.

If there exists a myopic-farsighted improving path from the strategy profile $a$ to $a^{\prime}$ we write $a \longrightarrow a^{\prime}$. The set of all strategy profiles that can be reached from $a$ by a myopic-farsighted improving path is denoted by $h(a)=\left\{a^{\prime} \in A \mid a \longrightarrow a^{\prime}\right\}$. Our notion of myopic-farsighted improving path is similar to the one first developed by Herings et al. (2020) for two-sided matching problems. ${ }^{13}$ When all players are myopic, this notion of myopic-farsighted improving path is similar to the notion of improving path proposed by Jackson and Watts (2002b) for network formation.

Example 4 Consider again the network depicted in Fig. 2. All players are myopic. Players 6 and 7 are stubborn while all other players are moderate. Player 6 is stubborn for project $p$ while player 7 is stubborn for project $q$. Both projects have low thresholds: $t^{p}=t^{q}=1$. Take the profile $a^{0}=(q, q, p, p, p, p, q)$ where players 3 , 4 and 5 choose project $p$ and players 1 and 2 select $q$. An example for an improving path from this profile is the following one. Player 2 has only one neighbor (player 1) with the same choice as her and three neighbors who choose project $p$. So it is an improvement for her to select $p$ as well. From this profile $a^{1}=(q, p, p, p, p, p, q)$

13 Herings et al. (2020) define the myopic-farsighted stable set for two-sided matching problems, while Luo et al. (2021) investigate the myopic-farsighted stable set in general network formation problems. 
there is a second step towards $a^{2}=(p, p, p, p, p, p, q)$. Since player 2 is now choosing project $p$, player 1 is not coordinating with any neighbor. She is better off if she switches her choice to project $p$. All players are myopic and consider the immediate improvement they get from changing their action. From $a^{2}=(p, p, p, p, p, p, q)$ we have $h\left(a^{2}\right)=\varnothing$. This is different if we consider farsighted players.

Suppose now that player 5 becomes farsighted. Start with the profile $a^{0}=$ $(p, p, p, p, p, p, q)$ where player 5 is coordinating with one of her two neighbors. From this profile there is an improving path initiated by player 5 . First, player 5 chooses project $q$ instead of $p$ which yields to the profile $a^{1}=(p, p, p, p, q, p, q)$. In this profile $a^{1}$ player 3 has now an incentive to deviate to project $q$ as well, because two of her three neighbors have selected project $q$. This yields to the profile $a^{2}=(p, p, q, p, q, p, q)$. We observe that in $a^{2}$ player 5 is coordinating with both of her neighbors. Since she is farsighted, she had the incentive to start the improving path, taking into account that player 3 will follow her example and deviate from project $p$ to $q$ afterwards. This makes player 5 in profile $a^{2}$ better off than in $a^{0}$, even though she did not immediately (in $a^{1}$ ) benefit from her initial change.

Only farsighted players anticipate action changes of the other players and compare their current utility with the utility they would get at the end of the improving path. Nash equilibrium requires strategy profiles to be immune to immediate deviations and does not capture that farsighted players anticipate the actions of other players and consider the end strategy profile that their deviations may lead to. In order to be stable, a strategy profile should be immune to deviations of both myopic and farsighted players. These considerations lead to the following definition of myopic-farsighted stable strategy profiles.

Definition 4 Consider $(g, t)$ as given. A strategy profile $a \in A$ is myopic-farsighted stable if it satisfies $h(a)=\emptyset$.

A strategy profile $a \in A$ is myopic-farsighted stable if there is no myopic-farsighted improving path leaving $a$. Let $\widehat{Z}(g, t)=\{a \in A \mid h(a)=\emptyset\}$ be the set of myopicfarsighted stable strategy profiles.

Let us first analyze some properties of the set of myopic-farsighted stable strategy profiles. The next proposition establishes that there are no cycles. That is, starting at $a$ there is no myopic-farsighted improving path that ends at $a$.

Proposition 5 Take any $a \in A$. We have $a \notin h(a)$.

Notice that if $a^{*} \in \mathcal{N}(g, t)$ is a Nash equilibrium, then there is no profitable individual deviation from $a^{*}$. Thus, $h\left(a^{*}\right)=\varnothing$ when all players are myopic, i.e. $N_{m}=M$. As a corollary of Proposition 5, we have that there always exist a myopicimproving path from any strategy profile $a \notin \mathcal{N}(g, t)$ to some Nash equilibrium strategy profile $a^{*} \in \mathcal{N}(g, t)$.

Corollary 1 Suppose that all players are myopic, $N_{m}=M$. Then, for any strategy profile a $\notin \mathcal{N}(g, t)$, there exists a Nash equilibrium strategy profile $a^{*} \in \mathcal{N}(g, t)$ such that $a^{*} \in h(a)$. 
From Proposition 5 and Corollary 1 we obtain the next theorem.

Theorem 2 Suppose that all players are myopic, $N_{m}=M$. The set of myopicfarsighted stable strategy profiles is equal to the set of Nash equilibria.

Theorem 2 states an important result as it shows the connection between the set of myopic-farsighted stable strategy profiles and the set of Nash equilibria. As long as all moderate players are myopic the sets are the same. Thus, if $N_{m}=M$ then $\widehat{Z}(g, t)=\mathcal{N}(g, t)$.

\subsection{Impact of farsightedness}

What happens to the set of myopic-farsighted stable strategy profiles when some myopic moderate players become farsighted or vice-versa? Let $\widehat{Z}(g, t, M, F)$ be the set of myopic-farsighted stable strategy profiles when $N_{m}=M \cup F$. Take some myopic (farsighted) player $i \in M(i \in F)$. We next show that the set of myopic-farsighted stable strategy profiles weakly decreases (increases) when a myopic (farsighted) player $i$ becomes farsighted (myopic). That is, $\widehat{Z}\left(g, t, M^{\prime}=M \backslash\{i\}, F^{\prime}=F \cup\{i\}\right) \subseteq$ $\widehat{Z}(g, t, M, F)$ and $\widehat{Z}(g, t, M, F) \subseteq \widehat{Z}\left(g, t, M^{\prime}=M \cup\{i\}, F^{\prime}=F \backslash\{i\}\right)$.

Theorem $3 \widehat{Z}\left(g, t, M^{\prime}=M \backslash\{i\}, F^{\prime}=F \cup\{i\}\right) \subseteq \widehat{Z}(g, t, M, F)$.

Theorem 3 implies that the set of stable profiles weakly decreases when a myopic player turns farsighted. From Theorem 1 we know that even though the set weakly decreases it will never become empty since $\widehat{Z}\left(g, t, M=\emptyset, F=N_{m}\right)=Z(g, t) \neq \varnothing$. One immediate conclusion from Theorem 3 is that, if players $i$ and $j$ become farsighted, the order in which they become farsighted does not matter for the set of stable strategy profiles. Take $\widehat{Z}(g, t, M, F)$ with $i, j \in M$. We have:

$$
\begin{aligned}
& \widehat{Z}(g, t, M \backslash\{i, j\}, F \cup\{i, j\}) \subseteq \widehat{Z}(g, t, M \backslash\{i\}, F \cup\{i\}) \subseteq \widehat{Z}(g, t, M, F) \\
& \widehat{Z}(g, t, M \backslash\{i, j\}, F \cup\{i, j\}) \subseteq \widehat{Z}(g, t, M \backslash\{j\}, F \cup\{j\}) \subseteq \widehat{Z}(g, t, M, F) .
\end{aligned}
$$

From Theorem 3 we obtain the following corollary.

Corollary 2 The set of myopic-farsighted stable strategy profiles when there are both myopic and farsighted players $\widehat{Z}(g, t, M, F)$ is a subset of the set of Nash equilibrium strategy profiles $\mathcal{N}(g, t)$. That is, $\widehat{Z}(g, t, M, F) \subseteq \mathcal{N}(g, t)$.

Thus, in our coordination game played on a fixed network, the set of myopicfarsighted stable strategy profiles when moderate players are both myopic and farsighted is a refinement of the set of Nash equilibrium strategy profiles.

Example 5 Consider again the network depicted in Fig. 2. Player 6 is stubborn for project $p$ while player 7 is stubborn for project $q$. Both projects have low thresholds: $t^{p}=t^{q}=1$. When all moderate players are myopic, there are six stable (or Nash equilibrium) strategy profiles:

$$
\begin{aligned}
& a^{1}=(p, p, p, p, p, p, q), a^{2}=(p, p, p, q, p, p, q), a^{3}=(p, p, q, p, q, p, q), \\
& a^{4}=(p, p, q, q, q, p, q), a^{5}=(p, q, q, q, q, p, q), a^{6}=(q, q, q, q, q, p, q) .
\end{aligned}
$$


Turning any of the players 2 to 4 into farsighted players, does not change the set of stable strategy profiles at all. But, if player 5 becomes farsighted, the first two profiles $a^{1}$ and $a^{2}$ are not stable any more. In those profiles player 5 deviates from project $p$ to project $q$ and takes into account that player 3 then will change to $q$ as well. Even when all players are farsighted the profiles $a^{3}, a^{4}, a^{5}$ and $a^{6}$ remain stable.

\subsection{Impact of stubbornness}

In the example of Fig. 2, there was one stubborn player for project $p$, one stubborn player for project $q$ and the remaining players were moderate. We can clearly observe that the set of myopic-farsighted stable strategy profiles does not only depend on the farsightedness of the moderate players, but also on the type of stubborn players and their position in the network.

Let $\widehat{Z}\left(g, t, N_{m}, N_{p}, N_{q}\right)$ be the set of myopic-farsighted stable strategy profiles when $N_{m}$ is the set of moderate players, $N_{p}$ is the set of stubborn players for project $p$ and $N_{q}$ is the set of stubborn players for project $q$. Let $\widehat{Z}\left(g, t, N_{m} \backslash\{i\}, N_{p} \cup\{i\}, N_{q}\right)$ be the set of myopic-farsighted stable strategy profiles when a moderate player $i$ becomes stubborn for project $p$. Obviously, we have that $\widehat{Z}\left(g, t, N_{m}, N_{p}, N_{q}\right) \subseteq$ $\left(\widehat{Z}\left(g, t, N_{m} \backslash\{i\}, N_{p} \cup\{i\}, N_{q}\right)\right) \cup\left(\widehat{Z}\left(g, t, N_{m} \backslash\{i\}, N_{p}, N_{q} \cup\{i\}\right)\right)$. If choosing a project $p$ is part of a stable strategy profile $a$ when player $i$ is moderate, then the strategy profile $a$ is still stable once player $i$ becomes stubborn for project $p$, while keeping fixed the type (moderate or stubborn) of the other players. That is, if $a \in \widehat{Z}\left(g, t, N_{m}, N_{p}, N_{q}\right)$ with $i \in N_{m}$ and $a_{i}=p$ then $a \in \widehat{Z}\left(g, t, N_{m} \backslash\{i\}, N_{p} \cup\{i\}, N_{q}\right)$. In addition, if there exists no stable strategy profile in which player $i$, while being moderate, chooses project $p$, new profiles become stable when player $i$ is stubborn for project $p$.

Example 6 Consider again the network depicted in Fig. 2. Assume that all players are myopic and thresholds are low, i.e. $t^{p}=t^{q}=1$. In the case that all players are moderate, there are eight different stable strategy profiles: $a^{1}=(p, p, q, p, q, p, q)$, $a^{2}=(p, p, q, q, q, p, q), a^{3}=(p, q, q, q, q, p, q), a^{4}=(p, p, p, p, p, p, p)$, $a^{5}=(q, q, p, q, p, q, p), a^{6}=(q, q, p, p, p, q, p), a^{7}=(q, p, p, p, p, q, p)$, $a^{8}=(q, q, q, q, q, q, q)$. Turning player 7 into a stubborn player for project $q$ keeps only those stable profiles in which player 7 chooses project $q$, namely: $a^{1}, a^{2}, a^{3}$ and $a^{8}$. In addition there are new stable strategy profiles: $a^{9}=(p, p, p, p, p, p, q)$, $a^{10}=(p, p, p, q, p, p, q)$ and $a^{11}=(q, p, p, p, p, q, q)$. We see that the profiles $a^{9}$ and $a^{11}$ are modifications from the profiles $a^{4}$ respectively $a^{7}$ : The actions of players 1 to 6 in profile $a^{9}\left(a^{11}\right)$ are the same as in $a^{4}\left(a^{7}\right)$ only the action of player 7 is different. In the strategy profile $a^{10}$ there is a change from at least two players compared to the profiles $a^{1}$ to $a^{4}$ and $a^{5}$ to $a^{8}$. If, in addition, we make player 6 stubborn for project $p$ we get the same six stable strategy profiles as in Example 5: $a^{1}, a^{2}, a^{3}, a^{9}, a^{10}$ and $a^{12}=(q, q, q, q, q, p, q)$. Again, turning a player into a stubborn player makes some strategy profiles unstable, while it stabilizes others.

On the other hand turning a player into a stubborn player does not always create new stable strategy profiles. Looking again at Example 5 where all players are myopic and player $6(7)$ is stubborn for $p(q)$, there are six stable strategy profiles. If we turn 
player 2 into a stubborn player for project $p$ only those four profiles where she chooses project $p$ remain stable. The same happens when we turn her into a stubborn player for project $q$. There are only two stable strategy profiles, the ones in which player 2 invests into project $q$.

Proposition 6 Suppose that the thresholds for both projects are low, $t^{p}=t^{q}=1$. If on all paths on the network $g$ between two players $i$ and $j$ there is a stubborn player, then the choices of player $i$ and $j$ are independent of each other.

When thresholds are low we can simplify our analysis with the help of Proposition 6. If there are two (or more) groups of players who are only connected to the other group(s) through paths that lead through at least one stubborn player we can split the graph into two (or more) components and analyze them separately. Proposition 6 can be a useful tool for a social planner trying to influence the choice between alternative projects in order to maximize coordination. According to this result, the social planner should first identify the different parts of the network that are connected only through stubborn players. Once identified the different parts of the network intermediated by stubborn players, the social planner should focus on stimulating the coordination in each of these parts separately rather than looking for coordination in the entire network. In other words, the social planner should not ignore the influence of the stubborn players on these different parts of the networks in order to promote coordination.

\subsection{Impact of the thresholds}

In addition to the types of players, the thresholds for both projects have an impact on the stability of the strategy profiles. If we increase the threshold for project $p$ from $t^{p}$ to $t^{p}+1$, then some strategy profiles may not be stable, while other strategy profiles may become stable. For instance, suppose that the threshold for project $q$ is low, $t^{q}=1$. If there is $a \in \widehat{Z}\left(g,\left(t^{p}, 1\right), N_{m}, N_{p}, N_{q}\right)$ such that $\#\left\{i \in N_{m} \mid a_{i}=p\right\}+\# N_{p}=t^{p}$, then $a \notin \widehat{Z}\left(g,\left(t^{p}+1,1\right), N_{m}, N_{p}, N_{q}\right)$. If there is a strategy profile in which the threshold for $p$ is exactly reached, then an increase of the threshold for this project will make it unstable since moderate players have incentives to switch to the low threshold project $q$.

\subsection{Some specific networks}

The stability of a strategy profile does not only depend on the thresholds and the type of players, but also on the network structure. We now analyze the set of stable strategy profiles for some common network configurations.

\subsubsection{Complete network}

In the case of the complete network, each player wants to coordinate with all other players. A moderate player wants to choose the project that gets chosen by the majority of the other players. This simplifies the analysis. 
Proposition 7 Consider the complete network $g^{N}$ with low thresholds, $t^{p}=t^{q}=1$. We have $\widehat{Z}\left(g^{N}, t, N_{m}, N_{p}, N_{q}\right) \subseteq\left\{a \in A \mid a_{i}=a_{j}\right.$ for all $\left.i, j \in N_{m}\right\}$.

Proposition 7 confirms that the complete network leads to a full coordination of the moderate players. There might be at most two stable profiles depending on the number of stubborn players for each project and on the number of farsighted players. But, in all of them the moderate players choose the same action. For instance, if $\# N_{p}>\# N_{q}-1$ and $\# F \geq \# M$ then $a \in A$ such that $a_{i}=p$ for all $i \in N_{m}$ is the unique myopic-farsighted stable strategy profile.

\subsubsection{Star networks}

In a star network the center of the star plays an important role. Depending on the farsightedness and the type (moderate or stubborn) of the player in the center, there can be different stable strategy profiles. Let player $i^{*}$ be the center of the star and suppose that project $q$ is the project with the most stubborn players $\left(\# N_{q}>\# N_{p}\right.$ ). Remember that $\# N_{m}+\# N_{r} \geq t^{r}$ for $r \in\{p, q\}$.

Proposition 8 Take a star network with $i^{*}$ being the center. Suppose that $t^{r}<n / 2$ for $r \in\{p, q\}$.

(i) If $i^{*} \in M$, then $\widehat{Z}(g, t)=\left\{a \in A \mid a_{i}=a_{j}\right.$ for all $\left.i, j \in N_{m}\right\}$;

(ii) If $i^{*} \in F$, \#F+\#N $\geq t^{q}$ and $\# N_{q}>\# N_{p}$, then $\widehat{Z}(g, t)=\left\{a \in A \mid a_{i}=q\right.$ for all $\left.i \in N_{m}\right\}$;

(iii) If $i^{*} \in N_{q}$ and $\# F+\# N_{q} \geq t^{q}$, then $\widehat{Z}(g, t)=\left\{a \in A \mid a_{i}=q\right.$ for all $\left.i \in N_{m}\right\}$.

Take any given strategy profile $a$. Since $t^{r}<n / 2$ for $r \in\{p, q\}$, the project chosen by the majority of players in $a$ is successful (without loss of generality project $q$ ).

(i) Hence, a myopic moderate player in the center has incentives to choose project $q$. Once she has adopted this project, all other moderate players who were choosing $p$ have now incentives to switch to $q$. Thus, all moderate players will fully coordinate on the same project in any stable strategy profile when the center is a myopic moderate player.

(ii) If the center is a farsighted moderate player (i.e. $i^{*} \in F$ ), then the choice of this player depends on the thresholds of the projects. If the project with the most stubborn

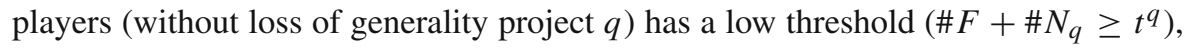
she chooses this project and anticipates that all other farsighted moderate players will follow her choice anticipating that all myopic moderate players will do the same afterwards. Through this choice she can coordinate with the highest possible amount of neighbors and the only stable strategy profile is that all moderate players choose project $q$ (i.e. full coordination among the moderate players).

(iii) If the center is a stubborn for project $q$ (without loss of generality) and her

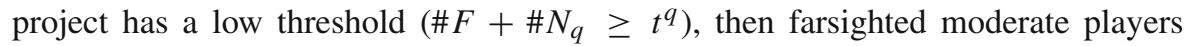
have incentives to choose $q$ anticipating that, once all of them have chosen project $q$, this project is for sure successful. Afterwards, all myopic moderate players have now incentives to adopt project $q$ too. Hence, the only stable strategy profile is that all moderate players choose project $q$ (i.e. full coordination among the moderate players). 


\subsubsection{Line networks}

In a line network we can sort players into two categories: the leaves (i.e. players who have only one neighbor) and the in-between players (i.e. players with two neighbors). We already know that in any stable profile moderate leaves choose the same project as their neighbor when thresholds are low. On the other hand, the in-between players can coordinate with one or two of their neighbors. When all players are myopic there exist many different stable profiles. In the case of low thresholds $\left(t^{p}=t^{q}=1\right)$, the line can be fragmented into groups of size 2 or larger. Players in each fragment choose the same project. If we consider a fragment of two moderate and myopic players, just turning one of them farsighted will increase the coordination. The farsighted player will choose the same project as her other neighbor (from a different fragment) and the myopic player will follow. In a fragment of three players we need to turn at least two of them farsighted in order to make players in the fragment coordinating with players from a neighboring fragment. Similarly, if the fragment consists of $x$ players, at least $x-1$ players have to become farsighted to achieve coordination with the neighboring fragments. The following proposition tells us when full coordination occurs on a line network.

Proposition 9 Consider a line network with only moderate players and with low thresholds, $t^{p}=t^{q}=1 . \widehat{Z}\left(g,(1,1), N_{m}=N\right)=\left\{a \in A \mid a_{i}=a_{j}\right.$ for all $\left.i, j \in N\right\}$ if and only if $\# \leq \leq 1$.

Thus, full coordination will always occur on a line network if and only if at most one player is myopic.

\section{Social planner}

We now look at the coordination problem from the point of view of a social planner. The objective of the social planner is to maximize the number of players who choose the same project. We consider two policy instruments. Either the social planner can socialize players by increasing the number of connections between players (i.e. linking players who are not yet directly connected) or the social planner can turn some myopic players into farsighted ones (through some educative plans). In the case of the complete network with low thresholds there is no need for an intervention of the social planner. The moderate players will always end up coordinating on the project chosen by the larger group of stubborn players. In the case of a star network with a myopic player in the center, the social planner just needs to turn this myopic player into a farsighted player for reaching the desired outcome.

From now on we analyze the use of those two instruments in the case of the circle network $g^{c}$. Remember that $\left\{i \in N \mid a_{i}=r\right\}$ is the set of players who choose project $r \in\{p, q\}, \# F$ is the number of farsighted players, and \#L $(g)$ is the number of added links in $g$. Both projects are assumed to have low thresholds, $t^{p}=t^{q}=1$. For instance, the social planner can either add a link $i j$ between $i$ and $j$ (if $i j \notin g$ ) or turn a myopic player $i$ into a farsighted one. Then, $M$ and $F$ become $M^{\prime}=M \backslash\{i\}$ and $F^{\prime}=F \cup\{i\}$. 
Without loss of generality, we consider coordination on project $p$. A strategy profile $a \in \widehat{Z}(g,(1,1))$ if and only if $a$ is such that either $a_{i}=p$ and $\left\{j \in N_{i}\left(g^{c}\right) \mid a_{j}=\right.$ $p\} \neq \emptyset$ or $a_{i}=q$ and $\left\{j \in N_{i}\left(g^{c}\right) \mid a_{j}=q\right\} \neq \emptyset$. Suppose that players along the circle are ordered: $\left\{i_{1}, i_{2}, \ldots, i_{n}\right\}$. A fragment of the circle is a subset of the ordered set of players, $\left\{i_{k}, i_{k+1}, \ldots, i_{k+l}\right\}$, such that each player in the fragment chooses the same project. Let $S_{r}^{l}\left(g^{c}, a\right)$ be a fragment $l$ of the circle network $g^{c}$ where players choose project $r$ in $a$. Notice that $\# S_{r}^{l}\left(g^{c}, a\right) \geq 2$. Let $S_{r}\left(g^{c}, a\right)=\cup_{l} S_{r}^{l}\left(g^{c}, a\right)=$ $\left\{j \in N_{m} \mid a_{j}=r\right\}$.

Proposition 10 Consider a circle network $g^{c}$. Suppose that all players are moderate, $N=N_{m}$, and both projects have low thresholds, $t^{p}=t^{q}=1$. Given some $a \in$ $\widehat{Z}\left(g^{c},(1,1)\right)$, in order to have full coordination on project $p$, the minimal requirements in terms of how many myopic players should become farsighted and/or how many links should be added are:

(i) $\# F=\#\left\{i \in N \mid a_{i}=q\right\}-1$, or

(ii) $\# L\left(g^{c}\right)=\#\left\{i \in N \mid a_{i}=q\right\}-1$, or

(iii) $\# F$ and $\# L=\#\left\{i \in N \mid a_{i}=q\right\}-1-\# F$, where $\# F<\#\left\{i \in N \mid a_{i}=q\right\}-1$.

Part (i) of Proposition 10 is in line with Proposition 9. The social planner has to turn mostly all moderate players who choose project $q$ into farsighted players in order to reach full coordination on project $p$. Part (ii) tells us that the social planner needs the same number of links (i.e. equal to the number of farsighted players needed for full coordination) in order to make the network denser and end up in full coordination. For instance, consider a fragment of two players choosing $q$. If both players are myopic, a unilateral change to project $p$ will not increase her utility. However, as soon as one of the players has an additional connection to a player choosing $p$, she is better off by switching her action to $p$, because now she has two neighbors choosing project $p$ versus one choosing $q$. If the fragment includes three players, adding two links between the two peripheral players (in this fragment) and two players choosing the opposite project $p$ makes them to adopt project $p$. Obviously, the central player (in this fragment) joins them afterwards. Part (iii) of Proposition 10 is about the joint implementation of both instruments: turning myopic players into farsighted ones and creating links between players with opposite projects. The two instruments act as substitutes since increasing the number of farsighted players reduces the needed number of links and vice versa. Obviously, those numbers are related to the total number of players who choose $q$.

To study the impact of stubbornness on the policies adopted by social planner, we now introduce one stubborn player for project $q$ into the circle network $g^{c}$. Remember that the presence of stubborn players for project $p$ does not affect the decision of the social planner as long as the objective of the social planner is to coordinate on project $p$.

Proposition 11 Consider a circle network $g^{c}$. Suppose that all players are moderate, $N=N_{m}$, except one player who is stubborn for project q, i.e. $N=N_{m} \cup N_{q}$ with $N_{q}=\left\{i^{q}\right\}$. Both projects have low thresholds, $t^{p}=t^{q}=1$. Given some $a \in \widehat{Z}\left(g^{c},(1,1)\right)$, in order to have full coordination on project $p$ among moderate players, the minimal requirements in terms of how many links should be added and how many myopic players should become farsighted are: 
(i) $\# L=\#\left\{i \in N \mid a_{i}=q\right\}-1$, or

(ii) $\# F$ and $\# L=\#\left\{i \in N \mid a_{i}=q\right\}-1-\# F$, where $0<\# F \leq \#\left\{i \in N \mid a_{i}=\right.$ $q\}-1-\#\left\{j \in N_{i} q\left(g^{c}\right) \mid a_{j}=q\right\}$.

Proposition 11 tells us that, once there is one player who is stubborn for the opposite project, the social planner cannot reach full coordination among the moderate players by only turning myopic players into farsighted ones. ${ }^{14}$ Consider any fragment with three players choosing $q$. If one of the players is stubborn, then even though the other two players are farsighted, they do not change their actions. Indeed, they know that their neighbor, the stubborn player, always chooses project $q$, and they would end up with the same utility if they switch to project $p$. On the contrary, if the social planner links each moderate player who is a neighbor of the stubborn player to some player choosing project $p$, then both players would now have incentives to switch to $p$ once their moderate neighbors have all chosen $p$. So, it suggests that adding links or promoting socialization can overcome some drawbacks of stubbornness in the society. Allowing players to communicate with a larger number of players reduces the likelihood of miscoordination, even in the presence of stubborn players. This argument is in line with Proposition 7 that confirms that the complete network leads to full coordination of the moderate players.

When there is no stubborn player for project $q$ (Proposition 10), the order for implementing both instruments (turning a myopic player into farsighted and/or adding a link) does not matter. However, in the presence of a stubborn player for project $q$, turning myopic players into farsighted ones without adding links first cannot lead to full coordination among the moderate players. Thus, a fraction of links has to be established first to provide the right incentives to the farsighted players for switching to project $p$.

The analysis about the circle network stresses the complexity of looking at the optimal intervention of the social planner for avoiding miscoordination among the moderate players. Moreover, it illustrates that a very good knowledge of the network is necessary for implementing efficiently policy instruments to favor full coordination on some project.

Let us illustrate the relevance of these results in social networks that can exhibit homophily or segregation. Cultural diversity and conflicting social identities are an important source of political and social tensions, declining solidarity and foregone economic opportunities. A population can be partitioned in different communities, according to ethnicity, language, religion, social class, political ideology, etc. The degree of identification with the communities we belong to likely affects our social interaction with people from other communities. In particular, people may be homophilic in the sense that they do not want to associate with strong partisans of another community, e.g., because this may result in conflict/unpleasant arguments. Consider a social planner facing two different communities and trying to influence them in order to adopt the same social norm. Then, in the absence of strong partisans (stubborn players) in these communities, educating a certain number of individuals to become farsighted could overcome homophily and reach the social planner's objec-

14 However, if there are only stubborn players for the project the social planner wants to coordinate on, then Proposition 10 applies. 
tive. However, if strong partisans are present in both communities, the social planner should first initiate a socialization policy (adding links between the different groups) before educating people to become farsighted.

\section{Conclusion}

We have studied a coordination game on a fixed connected network where players have to choose between two projects. Some players are moderate (i.e. they are exante indifferent between both project) while others are stubborn (i.e. they always choose the same project). Benefits for moderate players are increasing in the number of neighbors who choose the same project. In addition, players are either farsighted or myopic. Farsighted players anticipate the reactions of others while myopic players do not. We have shown that, when all players are farsighted, full coordination among the moderate players is reached except if there are stubborn players for both projects. When all players are myopic the set of stable strategy profiles coincides with the set of Nash equilibria of the coordination game. When the population is mixed, the set of stable strategy profiles is a refinement of the set of Nash equilibrium strategy profiles. Turning myopic players into farsighted ones eliminates little by little the inefficient Nash equilibria. In fact, when some myopic players become farsighted, efficiency measured by the sum of the utilities weakly improves. We have restricted the analysis to two projects. However, all our main results (e.g. Theorems 1, 2, 3, Propositions 1, 2) are robust to more projects. Finally, we have considered a social planner who can improve coordination by means of two policy instruments: adding links to the network (socialization) and/or turning myopic players into farsighted ones (education). The objective of the social planner is to maximize the number of players who choose the same project. If there is at most one type of stubborn players, this objective does not conflict with the maximization of the sum of utilities. However, if there are stubborn players for both projects, a tension may arise. Indeed, a group of players located in one part of the network may be better off by locally coordinating on some project, while another group of players located in another part of the network would be better off by locally coordinating on the other project. In this case, the only alternative for the social planner to reach full coordination would be to educate stubborn players to be moderate.

Acknowledgements Ana Mauleon and Vincent Vannetelbosch are, respectively, Research Director and Senior Research Associate of the National Fund for Scientific Research (FNRS). Financial support from the MSCA ITN Expectations and Social Influence Dynamics in Economics (ExSIDE) Grant no 721846 (1/9/2017-31/8/2020), from the Belgian French speaking community ARC project 15/20-072 of Saint-Louis University - Brussels, and from the Fonds de la Recherche Scientifique-FNRS research grant T.0143.18 is gratefully acknowledged. We acknowledge the editor and two anonymous referees for their suggestions and helpful comments.

Open Access This article is licensed under a Creative Commons Attribution 4.0 International License, which permits use, sharing, adaptation, distribution and reproduction in any medium or format, as long as you give appropriate credit to the original author(s) and the source, provide a link to the Creative Commons licence, and indicate if changes were made. The images or other third party material in this article are included in the article's Creative Commons licence, unless indicated otherwise in a credit line to the material. If 
material is not included in the article's Creative Commons licence and your intended use is not permitted by statutory regulation or exceeds the permitted use, you will need to obtain permission directly from the copyright holder. To view a copy of this licence, visit http://creativecommons.org/licenses/by/4.0/.

\section{Appendix}

Proof of Lemma 1 follows directly from Lemmas 2 and 3.

Lemma 2 Consider a strategy profile a with $a_{i} \neq a_{j}$ for some $i \neq j$. If at a, player $i$ prefers $a_{i}^{\prime}$ to $a_{i}$ (i.e. $\left.U_{i}\left(a_{i}^{\prime}, a_{-i}\right)>U_{i}\left(a_{i}, a_{-i}\right)\right)$ and at $\left(a_{i}^{\prime}, a_{-i}\right)$, player $j$ prefers $a_{j}^{\prime}$ to $a_{j}\left(i . e . U_{j}\left(a_{i}^{\prime}, a_{j}^{\prime}, a_{-i, j}\right)>U_{j}\left(a_{i}^{\prime}, a_{-i}\right)\right)$ with $a_{j}^{\prime} \neq a_{i}^{\prime}$, then it also holds that at $a$, player $j$ prefers $a_{j}^{\prime}$ to $a_{j}\left(\right.$ i.e. $\left.U_{j}\left(a_{j}^{\prime}, a_{-j}\right)>U_{j}\left(a_{j}, a_{-j}\right)\right)$.

Proof We prove that $U_{j}\left(a_{j}^{\prime}, a_{-j}\right) \geq U_{j}\left(a_{i}^{\prime}, a_{j}^{\prime}, a_{-i, j}\right)>U_{j}\left(a_{i}^{\prime}, a_{-i}\right) \geq U_{j}\left(a_{j}, a_{-j}\right)$ holds. Since $a_{i}^{\prime} \neq a_{j}^{\prime}, a_{i} \neq a_{i}^{\prime}$ and $a_{j} \neq a_{j}^{\prime}$, we have $a_{i}=a_{j}^{\prime}$ and $a_{i}^{\prime}=a_{j}$. Hence, $U_{j}\left(a_{j}^{\prime}, a_{-j}\right) \geq U_{j}\left(a_{i}^{\prime}, a_{j}^{\prime}, a_{-i, j}\right)$ and $U_{j}\left(a_{i}^{\prime}, a_{-i}\right) \geq U_{j}\left(a_{j}, a_{-j}\right)$ hold. If $i \in N_{j}(g)$ then the inequalities are strict.

Lemma 3 Consider a strategy profile a from which player $i$ wants to deviate to $a_{i}^{\prime}$ and some other player $j, i \neq j$, wants to deviate to $a_{j}^{\prime}$, with $a_{j}^{\prime} \neq a_{i}^{\prime}$, i.e. $U_{i}\left(a_{i}^{\prime}, a_{-i}\right)>$ $U_{i}\left(a_{i}, a_{-i}\right)$ and $U_{j}\left(a_{j}^{\prime}, a_{-j}\right)>U_{j}\left(a_{j}, a_{-j}\right)$. Once the deviations have taken place, if player $i$ deviates again to $a_{i}$ (because $\left.U_{i}\left(a_{i}, a_{j}^{\prime}, a_{-i, j}\right)>U_{i}\left(a_{i}^{\prime}, a_{j}^{\prime}, a_{-i, j}\right)\right)$, then player $j$ will never switch back to $a_{j}\left(\right.$ since $\left.U_{j}\left(a_{i}, a_{j}^{\prime}, a_{-i, j}\right)>U_{j}\left(a_{i}, a_{j}, a_{-i, j}\right)\right)$.

Proof of Proposition 1 Since there are no stubborn players, $N=N_{m}$. (1) Take $a$ such that $a_{i}=a_{j}$ for all $i, j \in N$. Then, $f(a)=\emptyset$ since $U_{i}(a) \geq U_{i}\left(a^{\prime}\right)$ for all $i \in N$, for all $a^{\prime} \in A$. (2) Take any $a^{\prime} \neq a^{*}$ where $a^{*}$ is such that $a_{i}^{*}=p$ for all $i \in N$. We will show that $a^{*} \in f\left(a^{\prime}\right)$ and so $f\left(a^{\prime}\right) \neq \emptyset$. Let $a^{0}=a^{\prime}$ and $a^{L}=a^{*}$. We build a farsighted improving path from $a^{0}$ to $a^{L}$ as follows. For $l=1, \ldots, L-1$, let

$$
a^{l+1}=\left\{\begin{array}{l}
a_{i}^{l+1}=a_{i}^{l}=p \text { for all } i \in\left\{j \in N \mid a_{j}^{l}=p\right\} \\
a_{i^{l}}^{l+1}=p \quad \text { for } i=i^{l} \text { with } a_{i^{l}}^{l}=q \text { and } N_{i^{l}}(g) \cap\left\{j \in N \mid a_{j}^{l}=p\right\} \neq \varnothing \\
a_{i}^{l+1}=a_{i}^{l}=q \text { for all } i \in\left\{j \in N \mid a_{j}^{l}=q\right\}, i \neq i^{l} .
\end{array}\right.
$$

The sequence of strategy profiles $a^{0}, \ldots, a^{L}$ is a farsighted improving path since along the sequence the player $i^{l}$ is such that $U_{i^{l}}\left(a^{L}\right)>U_{i^{l}}\left(a^{l}\right)$. Indeed, looking forward to $a^{*}$, player $i^{l}$ prefers $a^{*}$ to $a^{l}$ and so to switch to project $p$ anticipating that all players who haven't yet chosen $p$ will do it afterwards to finally reach the profile $a^{*}$. From (1) and (2) we have that $Z(g, t)=\left\{a \in A \mid a_{i}=a_{j}\right.$ for all $\left.i, j \in N\right\}$.

Proof of Proposition 2 Suppose $N=N_{m} \cup N_{p}$ and $N_{q}=\emptyset$. We have that all stubborn players for project $p$ choose $p$, i.e. $a_{i}=p$ for all $i \in N_{p}$. Project $p$ can be successful since $\# N_{m}+\# N_{p} \geq t^{p}$. (1) Take $a^{*}$ such that $a_{i}^{*}=p$ for all $i \in N$. Then, $f\left(a^{*}\right)=\emptyset$ since $U_{i}\left(a^{*}\right) \geq U_{i}\left(a^{\prime}\right)$ for all $i \in N_{m}$, for all $a^{\prime} \in A$. (2) Take any $a^{\prime} \neq a^{*}$ where $a^{*}$ is such that $a_{i}^{*}=p$ for all $i \in N$. We will show that $a^{*} \in f\left(a^{\prime}\right)$ and so $f\left(a^{\prime}\right) \neq \emptyset$. Let 
$a^{0}=a^{\prime}$ and $a^{L}=a^{*}$. We build a farsighted improving path from $a^{0}$ to $a^{L}$ as follows. Notice that in $a^{0}$ we have $a_{i}^{0}=p$ for all $i \in N_{p}$. For $l=1, \ldots, L-1$, let

$a^{l+1}= \begin{cases}a_{i}^{l+1}=a_{i}^{l}=p & \text { for all } i \in\left\{j \in N \mid a_{j}^{l}=p\right\} \\ a_{i^{l}}^{l+1}=p & \text { for } i=i^{l} \text { with } a_{i^{l}}^{l}=q \text { and } N_{i^{l}}(g) \cap\left\{j \in N \mid a_{j}^{l}=p\right\} \neq \varnothing \\ a_{i}^{l+1}=a_{i}^{l}=q & \text { for all } i \in\left\{j \in N \mid a_{j}^{l}=q\right\}, i \neq i^{l} .\end{cases}$

The sequence of strategy profiles $a^{0}, \ldots, a^{L}$ is a farsighted improving path since along the sequence the moderate player $i^{l}$ is such that $U_{i^{l}}\left(a^{L}\right)>U_{i^{l}}\left(a^{l}\right)$. Indeed, looking forward to $a^{*}$, player $i^{l}$ prefers $a^{*}$ to $a^{l}$ and so to switch to project $p$ anticipating that all players who haven't yet chosen $p$ will do it afterwards to finally reach the profile $a^{*}$. From (1) and (2) we have that $Z(g, t)=\left\{a \in A \mid a_{i}=p\right.$ for all $i \in N\}=\{(p, p, p, \ldots, p, p, p)\}$.

Proof of Proposition 3 Suppose $N=N_{m} \cup N_{p} \cup N_{q}$. The threshold $t$ is such that $\# N_{m}+\# N_{r} \geq t^{r}$ for $r \in\{p, q\}$. Suppose now that $\#\left(N_{i}(g) \cap N_{p}\right)>\#\left(N_{i}(g) \cap N_{q}\right)$ for all $i \in N_{m}$. (1) Take $a^{*}$ such that $a_{i}^{*}=p$ for all $i \in N_{m}$. Take any $i \in N_{m}$. Since $\#\left(N_{i}(g) \cap N_{p}\right)>\#\left(N_{i}(g) \cap N_{q}\right)$ we have that $U_{i}\left(a^{*}\right) \geq U_{i}\left(a^{\prime}\right)$ for all $a^{\prime} \in A$. Hence, $f\left(a^{*}\right)=\varnothing$. (2) Take any $a^{\prime} \neq a^{*}$ where $a^{*}$ is such that $a_{i}^{*}=p$ for all $i \in N_{m}$. We will show that $a^{*} \in f\left(a^{\prime}\right)$ and so $f\left(a^{\prime}\right) \neq \varnothing$. Let $a^{0}=a^{\prime}$ and $a^{L}=a^{*}$. We build a farsighted improving path from $a^{0}$ to $a^{L}$ as follows. Notice that in $a^{0}$ we have $a_{i}^{0}=p$ for all $i \in N_{p}$ and $a_{i}^{0}=q$ for all $i \in N_{q}$. For $l=1, \ldots, L-1$, let

$a^{l+1}=\left\{\begin{array}{l}a_{i}^{l+1}=a_{i}^{l}=p \text { for all } i \in\left\{j \in N_{m} \mid a_{j}^{l}=p\right\} \\ a_{i^{l}}^{l+1}=p \quad \text { for } i=i^{l} \text { with } a_{i^{l}}^{l}=q \text { and } N_{i^{l}}(g) \cap\left\{j \in N \mid a_{j}^{l}=p\right\} \neq \emptyset \\ a_{i}^{l+1}=a_{i}^{l}=q \text { for all } i \in\left\{j \in N_{m} \mid a_{j}^{l}=q\right\}, i \neq i^{l} \\ a_{i}^{l+1}=a_{i}^{l}=q \text { for all } i \in N_{q} \\ a_{i}^{l+1}=a_{i}^{l}=p \text { for all } i \in N_{p}\end{array}\right.$

The sequence of strategy profiles $a^{0}, \ldots, a^{L}$ is a farsighted improving path since along the sequence the moderate player $i^{l}$ is such that $U_{i^{l}}\left(a^{L}\right)>U_{i^{l}}\left(a^{l}\right)$ because of $\#\left(N_{i}(g) \cap N_{p}\right)>\#\left(N_{i}(g) \cap N_{q}\right)$. Indeed, looking forward to $a^{*}$, player $i^{l}$ prefers $a^{*}$ to $a^{l}$ and to switch to project $p$ because she has more stubborn players for project $p$ than for project $q$ in her neighborhood and she anticipates that all moderate players who haven't yet chosen $p$ will do it afterwards to finally reach the profile $a^{*}$. From (1) and (2) we have that $Z(g, t)=\left\{a \in A \mid a_{i}=p\right.$ for all $i \in N_{m} \cup N_{p}, a_{i}=q$ for all $i \in N_{q}$.

Proof of Proposition 4 Suppose that $t^{r} \leq \# N_{r}+1$ for $r \in\{p, q\}$. Take some moderate player $j \in N_{m}$ such that (i) \# $\left(N_{j}(g) \cap N_{p}\right)>\#\left(N_{j}(g) \cap N_{q}\right)$ and (ii) on all paths from $j$ to $i \in\left\{l \in N_{m} \mid \#\left(N_{l}(g) \cap N_{q}\right)>\#\left(N_{l}(g) \cap N_{p}\right)\right\}$ there is some stubborn player $k \in N_{p}$. We will show that player $j$ chooses $a_{j}=p$ in any farsightedly stable strategy profile in $Z(g, t)$. That is, for any $a^{\prime}$ such that $a_{j}^{\prime}=q, f\left(a^{\prime}\right) \neq \emptyset$. Since on all paths from $j$ to $i \in\left\{l \in N_{m} \mid \#\left(N_{l}(g) \cap N_{q}\right)>\#\left(N_{l}(g) \cap N_{p}\right)\right\}$ there is some stubborn player $k \in N_{p}$, we have that on all paths from $j$ all players $l^{*}$ between $j$ and the first 
stubborn player on the path (if any) are moderate players who have more stubborn players for project $p$ than for project $q$ in their neighborhood. It follows that all those players $l^{*}$ and player $j$ (weakly) prefer the strategy profile $a^{\prime \prime}$ such that $a_{l}^{\prime \prime}=p$, $a_{j}^{\prime \prime}=p$ and $a_{i}^{\prime \prime}=a_{i}^{\prime}$ for all $i \in N_{m}, i \neq j, l^{*}$, to the strategy profile $a^{\prime}$. From $a^{\prime}=a^{0}$ we build a farsighted improving to $a^{\prime \prime}=a^{L}$ as follows. Take some player $l^{*}$ (or player $j)$ such that $a_{l^{*}}^{\prime}=q$ and $N_{l^{*}}(g) \cap\left\{i \in N \mid a_{i}^{\prime}=p\right\} \neq \emptyset$. This moderate player $l^{*}$ is such that $U_{l^{*}}\left(a^{L}=a^{\prime \prime}\right)>U_{l^{*}}\left(a^{0}=a^{\prime}\right)$ and so she switches to project $p$ anticipating that players in her neighborhood will do the same afterwards. Next some other player $l^{*}$ (or player $j$ ) such that $a_{l^{*}}^{\prime}=a_{l^{*}}^{0}=a_{l^{*}}^{1}=q$ and $N_{l^{*}}(g) \cap\left\{i \in N \mid a_{i}^{1}=p\right\} \neq \emptyset$. This moderate player $l^{*}$ is such that $U_{l^{*}}\left(a^{L}=a^{\prime \prime}\right)>U_{l^{*}}\left(a^{1}\right)$ and so she switches to project $p$ anticipating that players in her neighborhood will do the same afterwards; and so forth until we reach the strategy profile $a^{L}=a^{\prime \prime}$ where $a_{l^{*}}^{\prime \prime}=p$ and $a_{j}^{\prime \prime}=p$. Hence, any $a \in Z(g, t)$ is such that player $j$ chooses $a_{j}=p$.

Proof of Proposition 5 We show that $a \notin h(a)$. Suppose there exists a myopicfarsighted improving path $a^{0}, a^{1}, \ldots, a^{L}$ with $a=a^{0}$ and $a=a^{L}\left(a^{k} \neq a^{l}\right.$ for all $k, l \in\{1, \ldots, L-1\}$ ). Notice first that no farsighted player will initiate such a path. Only myopic players could move away from $a$. Let $i^{1}$ be the myopic player that deviates from $a^{0}$ to $a^{1}$, with $U_{i^{1}}\left(a_{i^{1}}^{1}, a_{-i^{1}}^{0}\right)>U_{i^{1}}\left(a_{i^{1}}^{0}, a_{-i^{1}}^{0}\right)$. Consider now the move of player $i^{2}$ from $a^{1}$ to $a^{2}$. If $a_{i^{2}}^{2}=a_{i^{1}}^{1}$, then player $i^{1}$ will not deviate back to $a_{i^{1}}^{0}$ since the support for the project she prefers is now even larger. Thus, as long as, along the path, the successive deviating players continue supporting project $a_{i^{1}}^{1}$, player $i^{1}$ will not deviate back to $a_{i 1}^{0}$ and then the end strategy profile of the improving path will never be equal to $a$.

Let $l$ be the first time along the path such that some myopic player $j$ deviates from $a^{l}$ to $a^{l+1}$, with $a_{j}^{l+1} \neq a_{i^{1}}^{1}=\cdots=a_{i^{l}}^{l}$ and $U_{j}\left(a_{j}^{l+1}, a_{i^{1}}^{1}, \ldots, a_{i^{l}}^{l}, a_{-j, i^{1}, \ldots, i^{l}}^{0}\right)>$ $U_{j}\left(a_{i^{1}}^{1}, \ldots, a_{i^{l}}^{l}, a_{-i^{1}, \ldots, i}^{0}\right)$. Then by Lemma 2, we also have that player $j$ prefers $a_{j}^{l+1}$ to $a_{j}^{0}=a_{j}^{1}=\cdots=a_{j}^{l}$ before players $i^{1}, \ldots, i^{l}$ deviates from $a_{i^{1}}^{0}, \ldots, a_{i^{l}}^{l-1}$ to $a_{i^{1}}^{1}=\cdots=a_{i^{l}}^{l} \neq a_{j}^{l+1}$. It holds that

$$
\begin{aligned}
U_{j}\left(a_{j}^{l+1}, a_{i^{1}}^{1}, \ldots, a_{i^{l-1}}^{l-1}, a_{i^{l}}^{l}, a_{-j, i^{1}, \ldots, i^{l}}^{0}\right)>U_{j}\left(a_{i^{1}}^{1}, \ldots, a_{i^{l}}^{l}, a_{-i^{1}, \ldots, i^{l}}^{0}\right), \\
U_{j}\left(a_{j}^{l+1}, a_{i^{1}}^{1}, \ldots, a_{i^{l-1}}^{l-1}, a_{-j, i^{1}, \ldots, i^{l-1}}^{0}\right)>U_{j}\left(a_{i^{1}}^{1}, \ldots, a_{i^{l-1}}^{l-1}, a_{-i^{1}, \ldots, i^{l-1}}^{0}\right), \\
\\
U_{j}\left(a_{j}^{l+1}, a_{-j}^{0}\right)>U_{j}\left(a_{j}^{0}, a_{-j}^{0}\right) .
\end{aligned}
$$

Notice that, in order for the path to end at $a$, players $i^{1}, \ldots, i^{l-1}, i^{l}$ should have incentives to deviate back from $a_{i^{1}}^{1}=\cdots=a_{i^{l}}^{l}$ to $a_{i^{1}}^{0}, \ldots, a_{i^{l}}^{l-1}$, respectively. Thus, assume that this is the case once player $j$ (and possibly other players that deviate later on to the same project as player $j$ ) deviates from $a^{l}$ to $a^{l+1}$, with $a_{j}^{l+1} \neq a_{i^{1}}^{1}=\cdots=$ $a_{i}^{l}$. But then, by Lemma 3, we have that player $j$ (and possibly some other player) will never deviate back to $a_{j}^{0} \neq a_{j}^{l+1}$. Hence, $a$ will never be reached. 
Suppose now that, at $l$, a farsighted player $j$ deviates from $a^{l}$ to $a^{l+1}$, with $a_{j}^{l+1} \neq$ $a_{i^{1}}^{1}=\cdots=a_{i^{l}}^{l}$ and $U_{j}\left(a_{j}^{L}, a_{-j}^{L}\right)>U_{j}\left(a_{i^{1}}^{1}, \ldots, a_{i^{l}}^{l}, a_{-i^{1}, \ldots, i^{l}}^{0}\right)$. Then, in order to induce player $i^{1}$ to deviate back to $a_{i}^{0}$, the following case has to be considered: $a_{j}^{0}=a_{i^{1}}^{1}=\cdots=a_{i^{l}}^{l}$. We then obtain

$$
\begin{aligned}
U_{j}\left(a_{j}^{0}, a_{-j}^{0}\right) & \leq U_{j}\left(a_{i^{1}}^{1}, a_{j}^{0}, a_{-j, i^{1}}^{0}\right) \leq \cdots \leq U_{j}\left(a_{i^{1}}^{1}, \ldots, a_{i^{l}}^{l}, a_{j}^{0}, a_{-j, i^{1}, \ldots, i^{l}}^{0}\right) \\
& <U_{j}\left(a_{j}^{L}, a_{-j}^{L}\right)=U_{j}\left(a_{j}^{0}, a_{-j}^{0}\right),
\end{aligned}
$$

a contradiction. Thus, no farsighted player along the path from $a$ to $a^{L}=a$ would be the first player deviating from $a^{l}$ to $a^{l+1}$, with $a_{j}^{l+1} \neq a_{i^{1}}^{1}=\cdots=a_{i^{l}}^{l}$.

Proof of Theorem $2(\Rightarrow)$ We show that the set of Nash equilibrium strategy profiles $\mathcal{N}(g, t)$ is a set of myopic-farsighted stable strategy profiles $\widehat{Z}(g, t)$. Let some $a^{*} \in$ $\mathcal{N}(g, t)$ be given. It holds by definition of the Nash equilibrium that for all $a^{*} \in \mathcal{N}(g, t)$ we have $h\left(a^{*}\right)=\emptyset$. Hence, all $a^{*} \in \mathcal{N}(g, t)$ are myopic-farsighted stable strategy profiles. $(\Leftarrow)$ We show that $\mathcal{N}(g, t)$ is the only set of myopic-farsighted stable strategy profiles $\widehat{Z}(g, t)$. Let some $a \notin \mathcal{N}(g, t)$ be given. By Corollary 1 , we have that there exists a Nash equilibrium strategy profile $a^{*} \in \mathcal{N}(g, t)$ such that $a^{*} \in h(a)$. Hence, a strategy profile that is not a Nash equilibrium cannot be a myopic-farsighted stable strategy profile. Thus, if $N_{m}=M$ then $\widehat{Z}(g, t)=\mathcal{N}(g, t)$.

Proof of Theorem 3 Proof by contradiction. Suppose there is a strategy profile $a^{\prime} \in$ $\widehat{Z}\left(g, t, M^{\prime}=M \backslash\{i\}, F^{\prime}=F \cup\{i\}\right)$ and $a^{\prime} \notin \widehat{Z}(g, t, M, F)$. This means that there is another profile $a=\left(a_{i}, a_{-i}\right)$ with $a_{-i}=a_{-i}^{\prime}$ such that $U_{i}(a)>U_{i}\left(a^{\prime}\right)$. So that the deviation from $a_{i}^{\prime}$ to $a_{i}$ is an immediate improvement for player $i$. Then, there is a myopic-farsighted improving path of length one from $a^{\prime}$ to $a$ when player $i$ is myopic, with $a \in h\left(a^{\prime}\right)$. But then, the previous myopic-farsighted improving path of length one from $a^{\prime}$ to $a$ is also a myopic-farsighted improving path of length one from $a^{\prime}$ to $a$ when player $i$ is farsighted. This implies that $a^{\prime}$ is not stable if $i$ is farsighted and contradicts our assumption.

Proof of Proposition 6 Let $i^{r}$ be a stubborn player for project $r$. Without loss of generality, suppose that $i, j \in N_{i r}(g)$ and there exists no other path between $i$ and $j$ (otherwise repeat the same arguments). Clearly $a_{i}$ is independent of $a_{i}$ and $a_{j}$, because $i^{r}$ always chooses $a_{i} r=r$. This implies that $a_{i}$ and $a_{j}$ are independent of each other, that $a_{k}$ for $k \in N_{i}(g) \cap N_{m}$ is independent of $a_{j}$, that $a_{l}$ for $l \in N_{j}(g) \cap N_{m}$ is independent of $a_{i}$ and so on.

Proofs of Proposition 7, Proposition 8, Proposition 9 are available upon request.

Proof of Proposition 10. Take $S_{q}^{l}\left(g^{c}, a\right)=\left\{i_{1}, i_{2}, \ldots, i_{K}\right\}$ where $K=\# S_{q}^{l}\left(g^{c}, a\right)$. (i) Since $i_{k} \in N_{i_{k+1}}\left(g^{c}\right)$ for all $i_{k}, i_{k+1} \in S_{q}^{l}\left(g^{c}, a\right)$, the fragment is a line. Hence, the proof of Proposition 9 applies here. (ii) Players $i_{1}$ and $i_{K}$ have one neighbor choosing project $q$ and one neighbor choosing project $p$. By adding a new link $i_{1} j$ where $a_{j}=p$, we have $U_{i_{1}}\left(p, a_{-i_{1}}\right)>U_{i_{1}}\left(q, a_{-i_{1}}\right)$ and then player $i_{1}$ switches to 
project $p$. Analogously for player $i_{K}$. Now players $i_{2}$ and $i_{K-1}$ have one neighbor choosing project $q$ and one neighbor choosing project $p$. We next proceed to add links among $i_{2}$ and $i_{K-1}$ with two other players choosing $p$. We continue adding links between players in the fragment (with neighbors choosing $q$ and $p$ ) and players choosing $p$ until there is a unique player $i_{k}$ in the fragment who has both neighbors choosing project $p$, and so she has incentives to choose $p$. Therefore, we need to add $\# S_{q}^{l}\left(g^{c}, a\right)-1$ links to obtain full coordination on project $p$. (iii) We repeat the previous case (ii) until there are two players $i_{k}, i_{k+1}$ in the fragment who have one neighbor choosing project $q$ and one neighbor choosing project $p$. Suppose that player $i_{k}$ is farsighted, $\# F=1$, she will switch to project $p$, anticipating that the myopic player $i_{k+1}$ will join project $p$ as well. Therefore, with $\# F=1$ farsighted player and $\# L=\# S_{q}^{l}\left(g^{c}, a\right)-1-\# F=\# S_{q}^{l}\left(g^{c}, a\right)-2$ links we obtain the full coordination in the fragment. Following the same algorithm, if we increase the number of farsighted players $\left(\# F<\# S_{q}^{l}\left(g^{c}, a\right)-1\right)$ in the fragment, we could reduce the number of links needed for full coordination within the fragment.

Proof of Proposition 11 is available upon request.

\section{References}

Allouch N (2015) On the private provision of public goods on networks. J Econ Theory 157:527-552

Bramoullé Y (2007) Anti-coordination and social interactions. Games Econ Behav 58:30-49

Bramoullé Y, Lopez-Pintado D, Goyal S, Vega-Redondo F (2004) Network formation and anti-coordination games. Int J Game Theory 33:1-19

Bramoullé Y, Kranton R (2007) Public goods in networks. J Econ Theory 135:478-494

Chwe MS (1994) Farsighted coalitional stability. J Econ Theory 63:299-325

Dutta B, Ghosal S, Ray D (2005) Farsighted network formation. J Econ Theory 122:143-164

Goyal S (2007) Connections: an introduction to the economics of networks. Princeton University Press, Princeton

Goyal S, Vega-Redondo F (2005) Network formation and social coordination. Games Econ Behav 50:178207

Herings PJJ, Mauleon A, Vannetelbosch V (2009) Farsightedly stable networks. Games Econ Behav 67:526541

Herings PJJ, Mauleon A, Vannetelbosch V (2019) Stability of networks under horizon- $K$ farsightedness. Econ Theor 68:177-201

Herings PJJ, Mauleon A, Vannetelbosch V (2020) Matching with myopic and farsighted players. J Econ Theory 190:105125

Jackson MO (2008) Social and economic networks. Princeton University Press, Princeton

Jackson MO, Storms E (2019) Behavioral communities and the atomic structure of networks. Stanford University, Mimeo

Jackson MO, Watts A (2002a) On the formation of interaction networks in social coordination games. Games Econ Behav 41:265-291

Jackson MO, Watts A (2002b) The evolution of social and economic networks. J Econ Theory 106:265-295

Kirchsteiger G, Mantovani M, Mauleon A, Vannetelbosch V (2016) Limited farsightedness in network formation. J Econ Behav Organ 128:97-120

Leister CM, Zenou Y and Zhou J (2021) Social connectedness and local contagion. Rev Econ Stud (Forthcoming)

Luo C, Mauleon A, Vannetelbosch V (2021) Network formation with myopic and farsighted players. Econ Theor 71:1283-1317

Manea M (2021) Bottleneck links, essential intermediaries and competing paths of diffusion in networks. Theor Econ 16:1017-1053 
Mauleon A, Vannetelbosch V (2016) Network formation games. In: Bramoullé Y, Galeotti A, Rogers BW (eds) The Oxford handbook of the economics of networks. Oxford University Press, Oxford

Morris S (2000) Contagion. Rev Econ Stud 67:57-78

Page FH Jr, Wooders M (2009) Strategic basins of attraction, the path dominance core, and network formation games. Games Econ Behav 66:462-487

Ray D, Vohra R (2015) The farsighted stable set. Econometrica 83:977-1011

Ray D, Vohra R (2019) Maximality in the farsighted stable set. Econometrica 87:1763-1779

Sakovics J, Steiner J (2012) Who matters in coordination problems? Am Econ Rev 102:3439-3461

Publisher's Note Springer Nature remains neutral with regard to jurisdictional claims in published maps and institutional affiliations. 